\title{
RANDOM VARIATIONAL INEQUALITIES IN THE ABSENCE OF SAMPLE PATH CONTINUITY
}

\author{
S.A. ELSANOUSI \\ King Saud University \\ Department of Mathematics \\ College of Science \\ P.O. Box 2455 \\ Riyadh 11451, Saudi Arabia
}

(Received July, 1994; revised November, 1994)

\begin{abstract}
In this paper we investigate the existence of solutions to stochastic variational inequalities under conditions that are not imposed sample-path-wise.
\end{abstract}

Key words: Random Variational Inequalities, Brownian Motion, Ito Integral.

AMS subject classification: $35 \mathrm{~J} 85,49 \mathrm{~J} 40,47 \mathrm{~A} 68$.

\section{Introduction}

In [9], Noor and Elsanousi introduced the notion of a random variational inequality and demonstrated the existence of a solution upon assuming the random operator under consideration possesses strongly monotone and Lipschitz sample paths. This requirement makes it possible to view the random operator as a family of deterministic operators (indexed by the sample space), each having the kind of properties encountered in the theory of deterministic variational inequalities. We propose here an alternative set of sufficient conditions for the existence of a solution, under which the metric and probabilistic properties of the operator are inextricably linked.

We begin by introducing our notation and recalling a few basic definitions.

A probability space is a triplet $(\Omega, \mathcal{F}, P)$ where $\Omega$ is a non-empty set, $\mathscr{F}$ is a $\sigma$-algebra of subsets of $\Omega$, and $P$ is a probability measure on $\mathscr{F}$. A subset $\Lambda \subset \Omega$ is said to be null if there exists an $F \in \mathscr{F}$ with $\Lambda \subset F$ and $P(F)=0$. The space $(\Omega, \mathscr{F}, P)$ is complete if $\mathscr{F}$ contains all the null sets.

By the Borel $\sigma$-algebra of a Banach space $G$, we mean the smallest $\sigma$-algebra $\mathfrak{B}(G)$ containing all open sets. A map $f: \Omega \rightarrow T$ is said to be measurable if $f^{-1}(B) \in \mathscr{F}$ whenever $B \in \mathfrak{B}(G) . f$ is weakly measurable if $\phi \circ f: \Omega \rightarrow R$ is measurable for every $\phi \in G^{*}$, the dual of $G$. It is well known that separability of $G$ entails equivalence of measurability and weak measurability (see [13]). $\mathcal{L}^{0}(\Omega, \mathcal{F}, P, G)$ denotes the totality of measurable maps $f: \Omega \rightarrow G$. Two elements, $f$ and $g$ in $\mathcal{L}^{0}(\Omega, \mathcal{F}, P, G)$, are equivalent $(\bmod 0)$ if $\{w: f(w) \neq g(w)\}$ is null. The space of equivalence classes of this relation is denoted by $L^{0}(\Omega, \mathcal{F}, P, G)$. We shall, however, use the same symbol for a member of $\mathcal{L}^{0}(\Omega, \mathcal{F}, P, G)$ and the class it represents. $L^{p}(\Omega, \mathcal{F}, P, G)$ is the subset of $L^{0}(\Omega, \mathcal{F}, P, G)$ consisting of all $f$ such that $E|f|^{p}<\infty$ where $|\cdot|_{G}$ is the norm on $G$ and $E$ is the expectation with respect to $P$. 
A random operator on a Banach space $G$ is a map $T: \Omega \times G \rightarrow G$ such that $T(\cdot, x) \in$ $\mathscr{L}^{0}(\Omega, \mathcal{F}, P, G), \forall x \in G$. $T$ is said to be continuous (or to have continuous sample paths) if, for $w$ outside a null subset $\Lambda$, the map $T(\cdot, w): G \rightarrow G$ is continuous. $T$ is said to be measurable if $\forall B \in \mathfrak{B}(G), T^{-1}(B) \in \mathscr{F} \otimes \mathfrak{B}(G)$, the (product) $\sigma$-algebra generated by the rectangles $F \times B$ where $F \in \mathscr{F}$ and $B \in \mathscr{B}(G)$. If $G$ is separable, continuity of $T$ implies its measurability.

Throughout this paper, $(\Omega, \mathcal{F}, P)$ is a complete probability space, $H$ is a Hilbert space with norm $|\cdot|_{H}$ and inner product $\langle,\rangle_{H} \cdot K$ is a closed convex subset of $H$ and $P_{K}$ is the projection of $H$ onto $K . \quad L^{p}(\Omega, F, P, K)$ denotes the space of classes in $L^{p}(\Omega, \mathscr{F}, P, H)$ which have a representative whose range lies in $K$. $\widetilde{P}_{K}: L^{0}(\Omega, \mathcal{F}, P, H) \rightarrow L^{0}(\Omega, \mathcal{F}, P, K)$ is defined by

$$
\left(\widetilde{P}_{K} f\right)(\omega)=P_{K}(f(\omega)) \quad \forall f, \forall \omega .
$$

We shall occasionally write $\widetilde{H}$ for $L^{2}(\Omega, \mathscr{F}, P, H)$ and view $H$ as a subspace of $\widetilde{H}$. $\widetilde{H}$ is a Hilbert space with $\langle f, g\rangle_{\widetilde{H}}=E\langle f(\cdot), g(\cdot)\rangle_{H}$.

\section{The Random Variational Inequality}

Let $T$ be a measurable random operator on $H$ and suppose $h \in \mathcal{L}^{0}(\Omega, \mathcal{F}, P, H)$. We seek to solve the following problem.

Problem 1: Find $f_{0} \in \mathscr{L}^{0}(\Omega, \mathscr{F}, P, K)$ such that $\forall y \in K$

$$
\left\langle T\left(\omega, f_{0}(\omega)\right), y-f_{0}(\omega)\right\rangle_{H} \geq\left\langle h(\omega), y-f_{0}(\omega)\right\rangle_{H}
$$

for almost all $\omega$.

The measurability of $T$ with respect to $\mathscr{F} \otimes \mathscr{B}(H)$ ensures that for any $f \in \mathcal{L}^{0}(\Omega, \mathscr{F}, P, H)$ the mapping $\omega \mapsto T(\omega, f(\omega))$ is also in $\ell^{0}(\Omega, \mathcal{F}, P, H)$ and we can, thereby, define an operator $\widetilde{T}: L^{0}(\Omega, \mathcal{F}, P, H) \rightarrow L^{0}(\Omega, \mathcal{F}, P, H)$ via

$$
(\tilde{T} f)(\omega)=T(\omega, f(\omega)) .
$$

If order in $L^{0}(\Omega, \mathscr{F}, P, H)$ is determined point-wise almost everywhere, Problem 1 can be restated as follows.

Problem 1: Find $f_{0} \in L^{0}(\Omega, \mathcal{F}, P, K)$ such that $\forall y \in K$

$$
\left\langle\widetilde{T} f_{0}, y-f_{0}\right\rangle_{H} \geq\left\langle h, y-f_{0}\right\rangle_{H} .
$$

If $T$ (considered as a process indexed by $H$ ) were assumed to have continuous sample paths, then sufficient condition could be imposed on $T$ itself to ensure the existence of solution to Problem 1 (cf. [9]). If, however, sample continuity is not stipulated, we shall need to place our conditions on $\tilde{T}$ rather than $T$.

We make the following assumptions.

There exists a closed linear subspace $V$ of $\widetilde{H}=L^{2}(\Omega, \Im, P, H)$, containing $H$ such that

$\left(C_{1}\right) \quad V$ is invariant under $\widetilde{T}$ and $\widetilde{P}_{K}$ (i.e., $\widetilde{T} V \subset V, \widetilde{P}_{K} V \subset V$ ).

$\left.\left(C_{2}\right) \quad \widetilde{T}\right|_{V}$ is Lipschitz, i.e., $\exists \beta>0$ such that $\forall f, g \in V$

$$
\|\widetilde{T} f-\widetilde{T} g\|_{\widetilde{H}} \leq \beta\|f-g\|_{\widetilde{H}} .
$$


$\left.\left(C_{3}\right) \quad \tilde{T}\right|_{V}$ is strongly monotone, i.e., $\exists \alpha>0$ such that $\forall f, g \in V$

$$
\langle\widetilde{T} f-\widetilde{T} g, f-g\rangle_{\widetilde{H}} \geq \alpha\|f-g\|_{\widetilde{H}}^{2} .
$$

$\left(C_{4}\right) \quad h \in V$.

Theorem 2.1: Suppose $V, \widetilde{T}, h$ satisfy the conditions $\left(C_{1}\right)-\left(C_{4}\right)$. Then there exists a unique $f_{0} \in L^{2}(\Omega, \mathcal{F}, P, K) \cap V$ with the property that, for $\omega$ outside a null set $\Lambda \in \mathcal{F}$ and $\forall y \in K$,

$$
\left\langle T\left(\omega, f_{0}(\omega)\right), y-f_{0}(\omega)\right\rangle_{H} \geq\left\langle h(\omega), y-f_{0}(\omega)\right\rangle_{H}
$$

Before proving the theorem, we record the following well-known characterization of the projection $P_{K}$.

Lemma 2.1: If $P_{K}$ is the projection of the Hilbert space $H$ onto the closed convex subset $K$, then $\forall x \in H, z=P_{K} x$ if and only if

$$
\langle z, y-z\rangle_{H} \geq\langle x, y-z\rangle_{H}, \forall y \in K .
$$

Proof of Theorem 2.1: Let $\rho>0$. On account of $\left(C_{1}\right)$ and $\left(C_{4}\right)$, we can define an operator $S: V \rightarrow V$ by requiring

$$
S f=\widetilde{P}_{K}\{f-\rho[\tilde{T} f-h]\} .
$$

We shall choose $\rho$ in such a way that $S$ becomes a contraction on $V$.

Since $P_{K}$ is non-expansive, we have $\forall f, g \in V$

$$
\begin{aligned}
& |S f(\omega)-S g(\omega)|_{H}^{2} \leq|f(\omega)-g(\omega)-\rho[\tilde{T} f(\omega)-\tilde{T} g(\omega)]|_{H}^{2} \\
& \quad=|f(\omega)-g(\omega)|_{H}^{2}+\rho^{2}|\tilde{T} f(\omega)-\widetilde{T} g(\omega)|_{H}^{2}-2 \rho\langle\tilde{T}(\omega)-\tilde{T} g(\omega), f(\omega)-g(\omega)\rangle_{H} .
\end{aligned}
$$

Thus, by $\left(C_{2}\right)$ and $\left(C_{3}\right)$, we deduce that

$$
\|S f-S g\|_{\widetilde{H}} \leq \sqrt{1+\rho^{2} \beta^{2}-2 \rho \alpha}\|f-g\|_{\widetilde{H}} .
$$

Choosing $0<\rho<\frac{2 \alpha}{\beta^{2}}$ ensures that $\sqrt{1+\rho^{2} \beta^{2}-2 \rho \alpha}<1$, and turns $S$ into a contraction on $V$.

Let $f_{1} \in H$ and set $f_{n+1}=S f_{n}$. The sequence $\left(f_{n}\right)$ converges in mean to a fixed point $f_{0} \in F$ of $S$. On account of $\left(C_{2}\right), \widetilde{T} f_{n} \rightarrow \widetilde{T} f_{0}$ in $V$ also. By arranging for a subsequence (if necessary), we may assume $f_{n} \rightarrow f_{0}$ and $\widetilde{T} f_{n} \rightarrow \widetilde{T} f_{0}$ almost surely. It thus follows that $f_{0} \in V \cap \mathcal{L}^{2}(\Omega, \mathcal{F}, P, K)$ and that there exists a null set $\Lambda \in \mathcal{F}$ such that $\forall \omega \notin \Lambda$

$$
\begin{gathered}
f_{0}(\omega)=S f_{0}(\omega) \\
\text { i.e., } f_{0}(\omega)=P_{K}\left[f_{0}(\omega)-\rho\left[\tilde{T} f_{0}(\omega)-h(\omega)\right]\right] .
\end{gathered}
$$

This, by Lemma 2.1 , is equivalent to the requirement that $\forall y \in K$

$$
\left\langle T\left(\omega, f_{0}(\omega)\right), y-f_{0}(\omega)\right\rangle \geq\left\langle h(\omega), y-f_{0}(\omega)\right\rangle .
$$

To verify uniqueness, let us suppose $g_{0} \in L^{2}(\Omega, \mathcal{F}, P, K)$ is such that, for $\omega$ outside a null set $\Gamma$, we have $\forall y \in K$ 


$$
\left\langle T\left(\omega, g_{0}(\omega)\right), y-g_{0}(\omega)\right\rangle \geq\left\langle h(\omega), y-g_{0}(\omega)\right\rangle .
$$

Suppose $\omega \notin \Lambda \cup \Gamma$. Then with $y=g_{0}(\omega)$, we have from (1) that

$$
\left\langle\tilde{T} f_{0}(\omega), g_{0}(\omega)-f_{0}(\omega)\right\rangle \geq\left\langle h(\omega), g_{0}(\omega)-f_{0}(\omega)\right\rangle ;
$$

and with $y=f_{0}(\omega)$ in (3), we have

$$
\left\langle\widetilde{T} g_{0}(\omega), f_{0}(\omega)-g_{0}(\omega)\right\rangle_{H} \geq\left\langle h(\omega), f_{0}(\omega)-g_{0}(\omega)\right\rangle_{H}
$$

Consequently,

$$
\left\langle\widetilde{T} g_{0}(\omega)-\tilde{T} f_{0}(\omega), g_{0}(\omega)-f_{0}(\omega)\right\rangle \leq 0 \quad \forall \omega \notin \Lambda \cap \Gamma
$$

Taking expectations, we get

$$
\left\langle\widetilde{T} g_{0}-\widetilde{T} f_{0}, g_{0}-f_{0}\right\rangle_{\widetilde{H}} \leq 0 .
$$

$\left(C_{3}\right)$ now guarantees that $g_{0}=f_{0}$ in $L^{2}(\Omega, \mathcal{F}, P, H)$.

Remarks: 1 . The conclusion of Theorem 2.1 is stronger than the mere existence of a solution in $V$ for Problem 1. However, the uniqueness argument presented is for this stronger type of solution.

2. In many situations $\widetilde{T}$ may prove to be quite elusive and hard to describe which makes the job of checking conditions $\left(C_{1}\right)-\left(C_{4}\right)$ a tough task. If $T$ possesses a natural extension $\widehat{T}$ to a closed subspace $V$ of $\widetilde{H}$, then we may content ourselves with a solution to a weaker formulation of Problem 1.

Theorem 2.2: Suppose $V$ is a closed subspace of $\widetilde{H}$ and $\widehat{T}: V \rightarrow V$ is such that $\forall x \in H$, $(\widehat{T} x)(\omega)=T(\omega, x)$. If conditions $\left(C_{1}\right)-\left(C_{4}\right)$ are satisfied with $\widehat{T}$ in place of $\widetilde{T}$, then there exists an $f_{0} \in V \cap L^{2}(\Omega, \mathcal{F}, P, K)$ such that, for $\omega$ outside a null set $\Lambda$, we have

$$
\left\langle\left(\widehat{T} f_{0}\right)(\omega), y-f_{0}(\omega)\right\rangle \geq\left\langle f_{0}(\omega), y-f_{0}(\omega)\right\rangle, \quad \forall y \in K .
$$

Proof: The proof is exactly the same as that of Theorem 2.1.

\section{An Example}

Let $I=[0,1]$ and $H=L^{2}(I, \mathscr{B}(I), \ell, R)$ with Lebesgue measure $\ell$. Suppose $(\Omega, \mathscr{F}, P$, $\left.\left(W_{t}\right)_{t \in I},\left(\mathcal{F}_{t}\right)_{t \in I}\right)$ is a standard Brownian motion. We denote by $M^{2 k}(I)$ the space of all $\left(\mathcal{F}_{t}\right)$ non-anticipative processes $f:[0,1] \times \Omega \rightarrow R$ satisfying $E \int_{0}^{1} f(s, \omega)^{2 k} d s<\infty$. We can and shall identify $M^{2 k}$ as $L^{2 k}([0,1] \times \Omega, \mathscr{\rho}, R, \ell \times P)$ for a suitable $\sigma$-algebra $\mathscr{\rho}$ or as a subspace of $L^{2 k}(\Omega, \mathcal{F}, P, H)$. For each $x \in H$, the process $(t, \omega) \mapsto\left(\int_{0}^{t} x(s) d W_{s}\right)(\omega)$ admits a continuous version, and we can thus define an operator $B: \Omega \times H \rightarrow C(I) \subset H$ by

$$
B(\omega, x)(t)=\left(\int_{0}^{t} x(s) d W_{s}\right)(\omega) .
$$


We verify first that an $(\mathscr{F} \otimes \mathfrak{B}(H), \mathfrak{B}(C(I)))$ version of $B$ can be chosen. This is a consequence of general theory developed by Cohn, Hoffmann and Jorgensen (see $[3,7,8]$ ). Indeed, for each

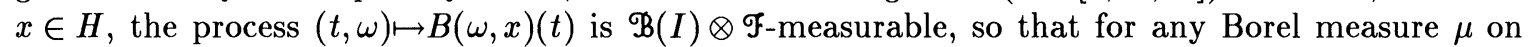

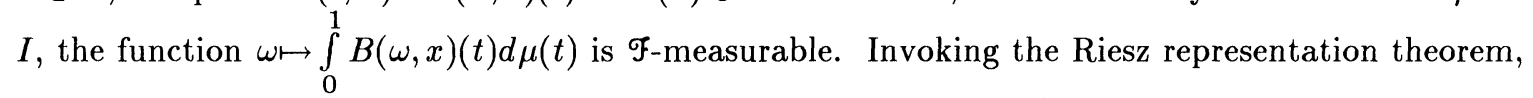
we deduce that $B(\cdot, x): \Omega \rightarrow C(I)$ is weakly measurable. Since $C(I)$ is separable, $B(\cdot, x)$ is in fact $(\mathscr{F}, \mathscr{B}(C(I)))$-measurable, and we can therefore view $B$ as a mapping from $H$ into $L^{0}(\Omega, \mathcal{F}, P, C(I))$. If we denote by $\|\cdot\|_{\infty}$ the uniform norm on $C(I)$, we have from the Martingale property of the Ito integral that

$$
E\left(\|B(\cdot, x)\|_{\infty}^{2}\right) \leq 4|x|_{H}^{2} .
$$

This ensures the continuity (and therefore measurability) of $B: H \rightarrow L^{0}(\Omega, \mathcal{F}, P, C(I))$. Since $H$ is complete, the existence of the said version follows from Cohn, Hoffman, and Jorgensen. We assume this version has been chosen.

We make the following observations.

$\left(O_{1}\right) \quad B$ is a strong random operator on $H$ that cannot be considered as a family of bounded linear operators on $H$ parameterized by $\omega \in \Omega$ (see Skorohod [11]).

$\left(\mathrm{O}_{2}\right) \quad$ Viewed as an $H$-valued stochastic process indexed by $H, B$ doesn't have continuous sample paths. To see this, we let $x_{n} \in H$ be defined by

$$
x_{n}=c_{n} \chi_{\left[o, \frac{1}{n}\right)}
$$

where $\chi$ is the indicator function and $c_{n}=\sqrt{\frac{n}{2 \log \log n}}$. Clearly $x_{n} \rightarrow 0$ in $H$. Were $B$ to have continuous sample paths, $\left|B\left(\omega, x_{n}\right)\right|_{H}$ would necessarily converge to zero almost everywhere. But

$$
B\left(\omega, x_{n}\right)(t)=\left\{\begin{array}{cc}
c_{n} W_{\frac{1}{n}}(\omega) & \text { if } t \geq \frac{1}{n} \\
c_{n} W_{t}(\omega) & \text { if } t<\frac{1}{n} .
\end{array}\right.
$$

Thus $\left|B\left(\omega, x_{n}\right)\right|_{H}^{2} \geq\left(\frac{n-1}{n}\right) c_{n}^{2} W_{\frac{1}{n}}^{2}(\omega)$.

Employing the law of Iterated Logarithm, we deduce that with probability 1, $\lim _{n \rightarrow \infty} \sup \left|B\left(\omega, x_{n}\right)\right|_{H} \geq 1$, which is the sought contradiction.

$\left(O_{3}\right)$ Let $f \in M^{2}(I)$. Then $\widetilde{B} f$ is defined by

$$
(\widetilde{B} f)(\omega)(t) \equiv B(\omega, f(\omega))(t)=\left(\int_{0}^{t} f(\omega, s) d W_{s}\right)(\omega) .
$$

It is tempting to confuse this with $\widehat{B} f(\omega(t))=\left(\int_{0}^{t} f(s) d W_{s}\right)(\omega)$, the stochastic integral of the process $f$. Equivalence of $\widetilde{B}$ and $\widehat{B}$ is assured for step and $C^{1}(I)$ processes $f$ but is at best conjectural for general $f \in M^{2}(I)$.

Now suppose $A: H \rightarrow H$ is Lipschitz (with constant $\beta_{0}$ ) and strongly monotone (with constant $\left.\alpha_{0}\right)$. Define, for $\alpha>\gamma>0$, the operator $T: \Omega \times H \rightarrow H$ by

$$
T(\omega, x)=A x+\gamma B(\omega, x) .
$$

Let $K=\{x \in H: x \geq 0$ a.e. $(\ell)$ on $I\}$. Then $K$ is a closed convex subset of $H$.

According to observation $\left(O_{2}\right), T$ does not have continuous sample paths (wrt $x$ ) and Problem 1 for $T$ and $K$ cannot be handled as in [9]. Observation $\left(O_{3}\right)$ indicates the difficulties in 
capturing the properties of $\widetilde{B}$ and so we resign ourselves to obtaining the solution of the weaker problem (as presented in Theorem 2.2).

Let $V=M^{2}(I)($ considered as a subspace of $\widetilde{H})$ and

$$
(\widehat{T} f)(\omega)(t)=A f(\omega)(t)+\gamma \widehat{B} f(\omega)(t)
$$

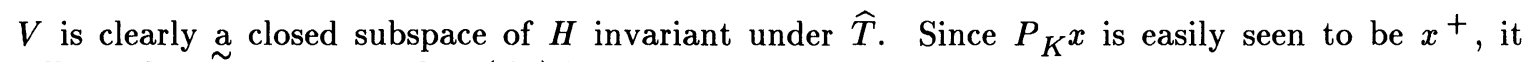
follows that $\widetilde{P}_{K} V \subset V$. Thus $\left(C_{1}\right)$ is satisfied.

$$
\begin{gathered}
\forall f, g \in V,|\widehat{T} f(\omega)-\widehat{T} g(\omega)|{ }_{H}^{2} \leq 2|A f(\omega)-A g(\omega)|{ }_{H}^{2}+2 \gamma^{2}|\widehat{B} f(\omega)-\widehat{B} g(\omega)|_{H}^{2} \\
\leq 2 \beta_{0}^{2}|f(\omega)-g(\omega)|_{H}^{2}+2 \gamma^{2}|\widehat{B} f(\omega)-\widehat{B} g(\omega)|^{2}
\end{gathered}
$$

so that

$$
\|\widehat{T} f-\widehat{T} g\| \underset{\widetilde{H}}{2} \leq 2\left(\beta_{0}^{2}+\gamma^{2}\right)\|f-g\|_{\widetilde{H}}^{2}
$$

and $\widehat{T}$ satisfies $\left(C_{3}\right)$ (with Lipschitz constant $\left.\sqrt{2\left(\beta_{0}^{2}+\gamma^{2}\right)}\right)$. Furthermore,

$$
\begin{gathered}
\langle(\widehat{T} f-\widehat{T} g)(\omega),(f-g)(\omega)\rangle_{H}=\langle A f(\omega)-A g(\omega), f(\omega)-g(\omega)\rangle_{H} \\
+\gamma\langle\widehat{B} f(\omega)-\widehat{B} g(\omega), f(\omega)-g(\omega)\rangle_{H}
\end{gathered}
$$

so that

$$
\langle\widehat{T} f-\widehat{T} g, f-g\rangle_{\widetilde{H}} \geq \alpha_{0}\|f-g\|_{\widetilde{H}}^{2}+\gamma E\langle\widehat{B} f-\widehat{B} g, f-g\rangle_{H}
$$

Now,

$$
\begin{aligned}
&\left|E\langle\widehat{B} f-\widehat{B} g, f-g\rangle_{H}\right|^{2}=\left[\int_{\Omega \times[0,1]}[f(\omega, t)-g(\omega, t)] \cdot[\widehat{B}(f-g)(\omega)(t)] d(P \times \ell)\right]^{2} \\
& \leq\|f-g\|_{\widetilde{H}}^{2} \cdot E \int_{0}^{1}[\widehat{B} f(t)-\widehat{B} g(t)]^{2} d t \\
&=\|f-g\|_{\widetilde{H}}^{2} \cdot \int_{0}^{1} E(\widehat{B} f(t)-\widehat{B} g(t))^{2} d t \\
&=\|f-g\|^{2} \int_{0}^{1}[f(s)-g(s)]^{2} d s d t \\
& \leq\|f-g\|_{H}^{4} .
\end{aligned}
$$

Thus $\langle\widehat{T} f-\widehat{T} g, f-g\rangle_{\widetilde{H}} \geq\left(\alpha_{0}-\gamma\right)\|f-g\|_{\widetilde{H}}^{2}$ and $\left(C_{4}\right)$ is verified (with $\alpha=\alpha_{0}-\gamma$ ).

From Theorem 2.2 we now conclude the existence an $f_{0} \in M^{2}(I) \cap L^{2}(\Omega, \mathcal{F}, P, K)$ and a null $\Lambda$ such that, for $\omega \notin \Lambda$, we have

$$
\left\langle\widehat{T} f_{0}(\omega), f_{0}(\omega)-y\right\rangle \geq\left\langle f_{0}(\omega), y-f_{0}(\omega)\right\rangle, \quad \forall y \in K .
$$




\section{References}

[1] Baiocchi, C. and Capelo, A., Variational and Quasi-variational Inequalities, John Wiley and Sons 1984.

[2] Bharucha-Reid, A.T., Random Integral Equations, Academic Press, New York 1972.

[3] Cohn, D.L., Measurable choice of limit points and the existence of separable and measurable processes, Z. Wahr. Verw. Geb. 22 (1972), 161-165.

[4] Dunford, N. and Schwartz, J.T., Linear Operators, Part I: General Theory, Interscience Publishers 1966.

[5] Gihman, I.I. and Skorohod, A.V., Stochastic Differential Equations, Springer-Verlag 1973.

[6] Hida, T., Brownian Motion, Springer-Verlag 1980.

[7] Hoffman-Jorgensen, Existence of measurable modifications of stochastic processes, $Z$. Wahr. Verw. Geb. 25 (1973), 205-207.

[8] Mohammed, S.E.A., Stochastic Functional Differential Equations, Research Notes in Mathematics 99, Pitman Books Limited 1984.

[9] Noor, M.A. and Elsanousi, S.A., Iterative algorithms for random variational inequalities, Pan Amer. Math. Journal 3 (1993), 39-50.

[10] Noor, M.A. and Elsanousi, S.A., Random nonlinear variational inequalities, Pan Amer. Math. Journal 4 (1994), 73-87.

[11] Skorohod, A.V., Random Linear Operators, Mathematics and its Applications, D. Reidel Publishing Company 1984.

[12] Tan, K., Tarafdar, E. and Yuan, X.Z., Random variational inequalities and applications to random minimization and nonlinear boundary problems, Pan Amer. Math. Journal 4 (1994), 55-71.

[13] Yosida, K., Functional Analysis, Springer-Verlag 1971. 


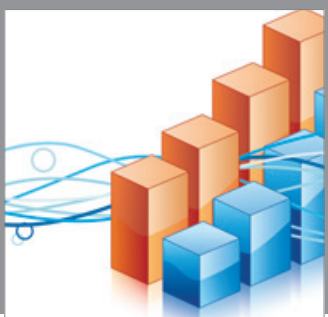

Advances in

Operations Research

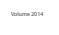

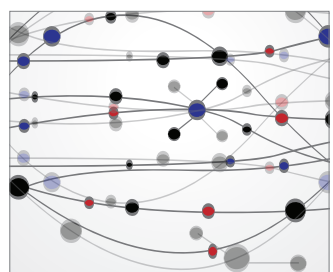

\section{The Scientific} World Journal
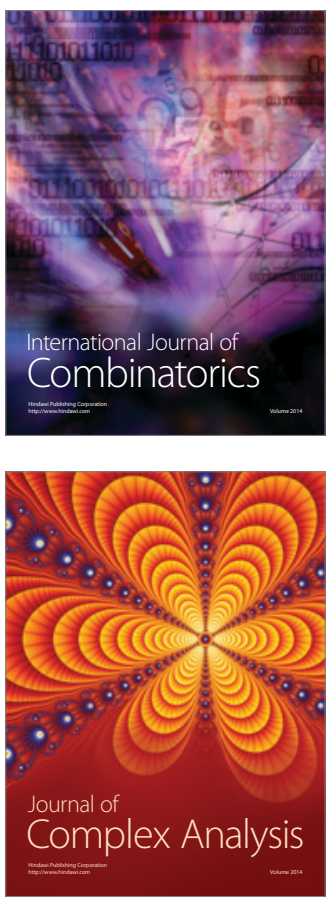

International Journal of

Mathematics and

Mathematical

Sciences
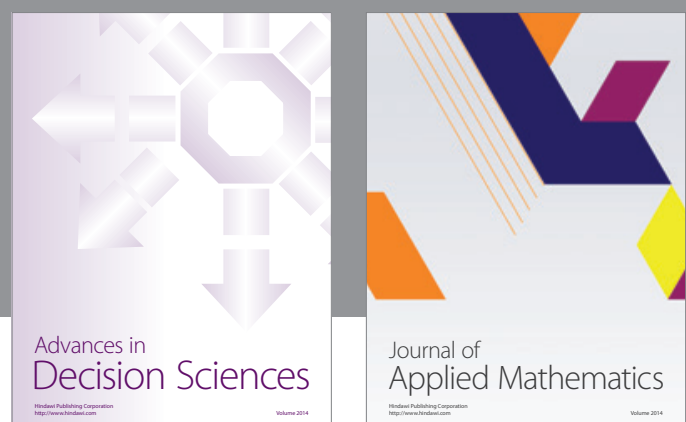

Journal of

Applied Mathematics
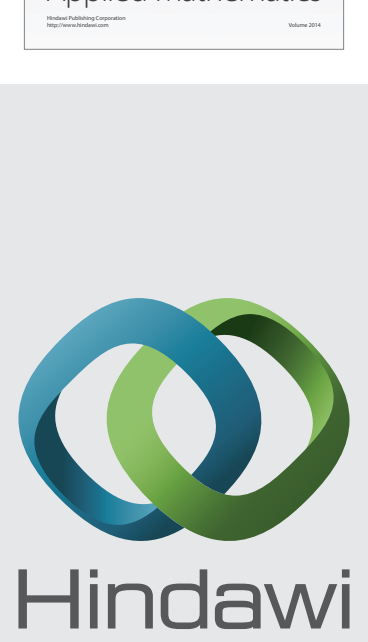

Submit your manuscripts at http://www.hindawi.com
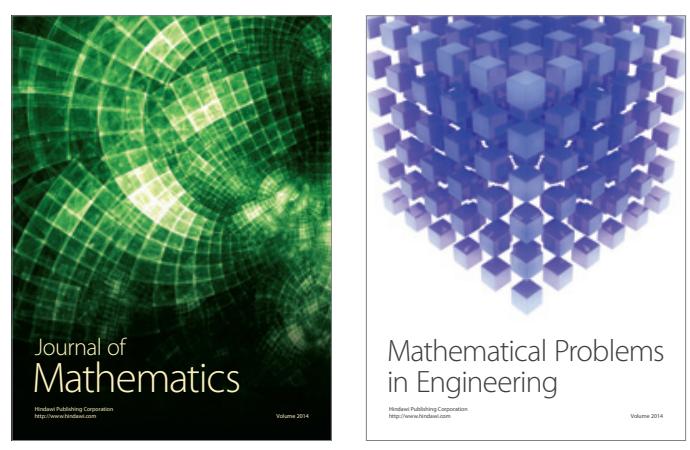

Mathematical Problems in Engineering
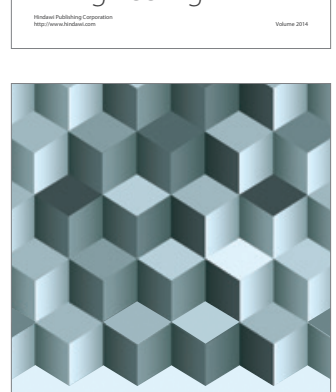

Journal of

Function Spaces
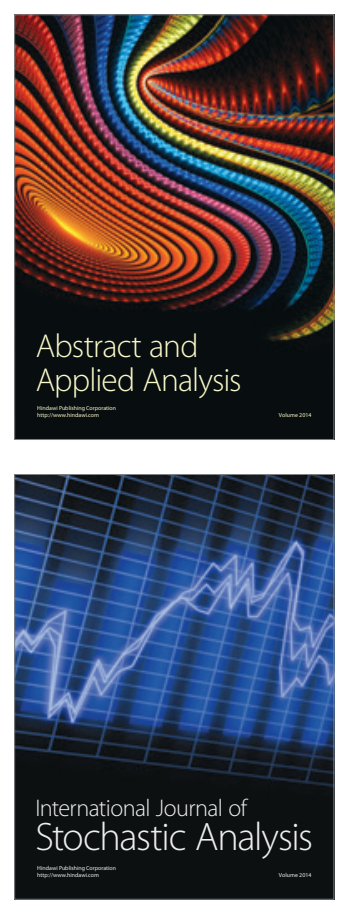

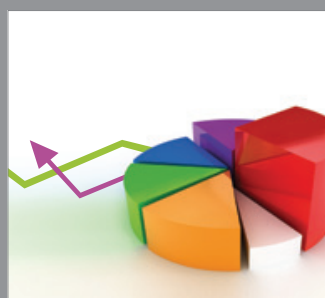

ournal of

Probability and Statistics

Promensencen
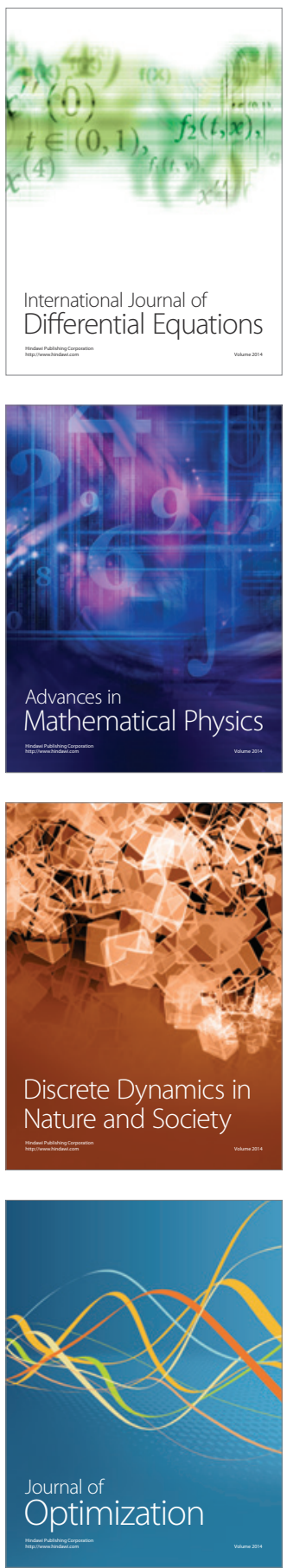